\title{
Haplo-block structure of Southern African village chicken populations inferred using genome-wide SNP data
}

\author{
K.S. Khanyile ${ }^{1,2}$, E.F. Dzomba ${ }^{2}$ and F.C. Muchadeyi ${ }^{1}$ \\ ${ }^{1}$ Biotechnology Platform, Agricultural Research Council, Onderstepoort, South Africa \\ 2University of KwaZulu-Natal, Discipline of Genetics, School of Life Sciences, \\ Pietermaritzburg, South Africa \\ Corresponding author: F.C. Muchadeyi \\ E-mail: MuchadeyiF@arc.agric.za \\ Genet. Mol. Res. 14 (4): 12276-12287 (2015) \\ Received February 6, 2015 \\ Accepted July 1, 2015 \\ Published October 9, 2015 \\ DOI http://dx.doi.org/10.4238/2015.October.9.16
}

ABSTRACT. This study investigated the haplo-block structure, haplotype sharing, and diversity in extensively raised chicken populations of Southern Africa. Two hundred ninety village chickens from Malawi $(N=30)$, South Africa ( $\mathrm{N}=132)$, and Zimbabwe $(\mathrm{N}=128)$ were included in the study, from which 649, 2104, and 2442 haplo-blocks were observed, respectively. The majority of haplo-blocks were smaller than $25 \mathrm{~kb}$ in size and only five blocks were more than $2000 \mathrm{~kb}$ in size. The low chromosomal coverage of haploblocks observed across the genome suggests that multiple recombination events fragmented the ancestral haplo-blocks into smaller sizes. Haploblock sharing was observed between populations with 2325 haplo-blocks common between Zimbabwe and Malawi and 2689 between South Africa and Zimbabwe. Haplotype sharing allows transferability of genomic tools between these extensively raised chicken populations of Southern Africa. The unique haplo-blocks could have originated from isolated evolution taking place in specific agro-ecological zones. Quantitative trait loci analysis revealed that genes related to body composition were spanned by these haplo-blocks. Body composition traits are important for village 
chicken populations, which have to harness poor quality feed obtained from the environment to meet their maintenance and production needs.

Key words: Haplo-block structure; Genetic diversity; SNPs; Village chickens

\section{INTRODUCTION}

The evolution of village chicken populations in Southern Africa and other developing countries is not clearly understood. It is assumed that communal farmers who raise village chickens do not impose selection pressures for breed development as is performed in the commercial sector. However, it is also hypothesized that these chickens are exposed to agro-ecological conditions that impose natural selection pressures. These pressures shape the gene pool of extensively raised livestock species (Muchadeyi et al., 2007a) and generate sub-populations that accumulate long ancestral haplotypes supporting isolated genetic change. Genetic variation is expected to be high in village chickens because they have not experienced artificial selection and are thought to be structured similarly to natural populations (Muchadeyi et al., 2006, 2007a,b; Mtileni et al., 2011). Genetic variation is also expected to be influenced by geographical isolation of these populations. Pockets of inbred chickens arise from low effective population sizes in each sub-population; this is because of uncontrolled mating systems as well as natural selection pressures from uncontrolled disease outbreaks, coupled with poor and fluctuating feed supplies (Li and Merilä, 2011).

Studies of linkage disequilibrium (LD) revealed low LD in village chicken populations, which is consistent with a high effective population size and limited utility of the Illumina iSelect chicken SNP60K bead chip in Southern African village chicken populations. The shift from sparsely distributed to high-density markers has made it possible to investigate haplo-block structures in many livestock species (Amaral et al., 2008; Megens et al., 2009; Qanbari et al., 2010) and in humans (Zhao et al., 2003). Several studies on haplotype diversity in livestock have been conducted since whole-genome sequences were made available, and the development of highdensity marker panels became feasible (Tishkoff and Verrelli, 2003; Zhao et al., 2003; LindbladToh et al., 2005; Amaral et al., 2008; Megens et al., 2009). Haplotype blocks are defined as the number (two or more) of alleles in loci that are closely linked and are transferred together (Zhao et al., 2003; Crawford and Nickerson, 2005). Haplo-blocks are more informative than single alleles in a genome because markers spanning haplotypes are more stable. Haplo-blocks can be useful for gene mapping during molecular breeding programs and for detecting disease variants. Haplotypes and haplo-blocks have been found to span across quantitative trait regions for traits of economic importance and for diseases variants. In population diversity studies, haplotype sharing amongst individuals can indicate the presence of conserved genomic regions that are a result of intensive and directional natural or artificial selection, and are therefore useful in fine mapping of quantitative trait loci (QTL) of economic importance.

Chickens are an avian species and have a unique genetic architecture that comprises macro-, intermediate-, and micro-chromosomes (Megens et al., 2009; Qanbari et al., 2010; Ensembl, 2013). Each chromosome set has a different genetic structure and composition, and it is characterized by different LD patterns. Studies on LD have suggested the presence of blocklike structures of markers that are distributed across the chicken genome (Megens et al., 2009). Genomic regions that undergo few recombination events form a block-like structure that can be shared among individuals within and between populations. Haplotype boundaries are assumed to be structured by recombination hot spots, while recombination cold-spots introduce variation within 
haplo-blocks (Gabriel et al., 2002; Zhao et al., 2003). The extent and distribution of haplo-blocks throughout the genome is considered a function of the genetic structure, genetic variation, and the demography of populations.

Genome-wide analysis of haplotypes and the distribution of haplo-blocks in chickens can help to identify regions that are associated with phenotypic traits or adaptive features. Haplotypes were investigated on selected genomic regions and chromosomes prior to the genomics era, mainly because of a lack of tools that could scan whole genomes. Analysis of selected regions made it difficult to compare populations that had been analyzed in different studies and on different genomic regions. Regardless of these limitations, such analyses added valuable information to our knowledge of the genetic architecture of populations. In the next generation sequencing era, genomics have used genome-wide haplo-block partitioning to reveal the degree of haplotype sharing and diversity within and among breeds (Amaral et al., 2008). Next generation sequencing and genome-wide SNP genotyping technologies have made large SNP datasets available that can be used to study most domestic livestock species. The first draft of the chicken genome was published in 2004 (International Chicken Genome Consortium, 2004) from which over 2.8 million SNPs have been discovered. The Illumina chicken iSelect $60 \mathrm{~K}$ SNP chip has been useful for studying LD as well as haplo-blocks partitioning in commercial (Qanbari et al., 2010) and traditional free-range chickens (Wragg et al., 2012).

There is no information on the haplo-block structure of extensively raised chicken populations in Southern Africa. These chicken populations are raised under scavenging village production systems, and the genetic structure of populations seems to be affected by natural selection pressures from the extreme environments as well as from inbreeding due to them being small sub-structured populations. LD analysis in these populations indicated that there are chromosomal differences in LD (Khanyile et al., 2015). The influence of recombination and the existence of haplo-blocks associated with the observed LD profile have not been investigated. An analysis of haplo-block structure in these extensively raised chickens will broaden our understanding of the utility of the available SNP panel for studying village chicken populations.

Therefore, the objective of this study was to screen for haplo-blocks and investigate haplotype structure, sharing, and diversity within and between village chicken populations of Southern Africa. The hypothesis that differences exist in the haplo-blocks of village chicken populations from different geographical origins due to isolated evolutionary processes was tested.

\section{MATERIAL AND METHODS}

\section{Animal populations}

Three hundred twelve village chickens were randomly sampled from ecotypes within three Southern African countries, South Africa (three ecotypes), Malawi (one ecotype), and Zimbabwe (three ecotypes). Collection of samples from each country followed the framework described previously by Muchadeyi et al. (2007) and Mtileni et al. (2011).

\section{SNP quality control and pruning}

SNP pruning was performed using PLINK v1.07 (Purcell et al., 2007 ) for SNPs that had a minor allele frequency of 0.05 , over $5 \%$ missing genotypes, that deviated from Hardy-Weinberg equilibrium (at $\mathrm{P}=0.001$ ), and for individuals with over $5 \%$ missing genotypes. Using these quality 
control criteria, 45,676, 46,905, and 44,667 markers were available for further analysis of the overall population from Malawi, Zimbabwe, and South Africa, respectively. Following quality control, 290 of 312 individuals from Malawi $(N=30)$, South Africa $(N=132)$, and Zimbabwe $(N=128)$ were used for downstream analysis.

\section{Haplo-block partitioning}

Haplo-blocks were estimated in PLINK v1.07 (Purcell et al., 2007 ), which uses default procedures from Haploview (Barrett et al., 2005) (http://www.broad.mit.edu/mpg/haploview/). Pairwise LD was calculated on an SNP distance of at least $10 \mathrm{~kb}$ for autosomal chromosomes except for chromosome 16, which had less than 20 markers. For block partitioning, -blocks function was used in PLINK using the default algorithm described by Gabriel et al. (2002) as implemented in Haploview. Regions were defined as haplo-blocks if the $95 \%$ confidence bound by $\mathrm{r}^{2}$ was between 0.7 and 0.98. The frequency of haplo-blocks was estimated in PLINK using the -hap-freq function. Blocks of different frequencies were estimated per chromosome for the blocks occurring at a frequency of $<0.1,0.1-0.25,0.25-0.5,0.5-0.75$, and 0.75-1.0.

The percentage of SNPs making up a haplo-block was calculated by dividing the number of SNPs within a block by the total number of SNPs used in the haplo-block partitioning, multiplied by 100 . The unique number of haplo-blocks was defined as the number of blocks that only occurred in one population.

\section{Haplotype diversity and QTL detection}

Haploview v4.2 (Barrett et al., 2005) was used to generate LD plots and to estimate the haplotype frequency within blocks per population. Chromosome 8 was selected for further analysis based on the high number of long haplo-blocks that had higher genome coverage (Table 1). We hypothesized that haplotype diversity was high on those chromosomes that could be harboring QTLs under selection for the adaptation of village chicken populations to the harsh production systems. On chromosome 8, haplo-blocks more than $50 \mathrm{~kb}$ in size were selected, and for each haplo-block the minimum number of SNPs was set at two. Haplotype diversity was considered as the number of haplotypes found within a haplo-block. The first and last position of SNP markers was used to search for possible QTLs spanned by the block for each chromosome using the Chicken QTL Database: http://www.animalgenome.org/cgi-bin/QTLdb/GG.

\section{RESULTS}

\section{Haplo-blocks characteristics per chromosome}

The mean number of haplo-blocks per chromosome was 100.9, and ranged from 13 on chromosome 25 to 402 on chromosome 1 (Table 1). The average length of haplo-blocks was $18.8 \mathrm{~kb}$, and ranged from 6.2 to $53.1 \mathrm{~kb}$. The longest haplotypes were observed on chromosome 8 and the shortest were on chromosome 25 . Chromosome coverage averaged $0.08 \mathrm{Mb}$, and high chromosome coverage was observed on chromosomes 8 and 11 and low coverage on chromosomes 3, 6, 9, 24, and 25 (Table 1). The majority of haplo-blocks were on macro-chromosomes (chromosomes 1-5) and fewer were on micro-chromosomes (chromosomes 16-28). Figure 1 shows the distribution of haplo-blocks of varying lengths across the different chromosomes. Table 2 shows the variation in 
haplo-block size per population, in which the majority of haplo-blocks were less than $10 \mathrm{~kb}$ followed by a significant proportion that ranged from 10 to $25 \mathrm{~kb}$ in size. The frequency of short haplo-blocks $(\leq 25 \mathrm{~kb})$ was consistently high across populations. Haplo-blocks larger than $1 \mathrm{Mb}$ were observed at a low frequency across all populations.

\begin{tabular}{|c|c|c|c|c|c|c|c|c|}
\hline Chromosome & Blocks & $\begin{array}{l}\text { Chromosome } \\
\text { coverage (\%) }\end{array}$ & $\begin{array}{c}\text { Mean block } \\
\text { length }(k b) \pm \mathrm{SD}^{*}\end{array}$ & $\begin{array}{l}\text { Median block } \\
\text { length }(k b) \pm S D\end{array}$ & SNPs (\%) & $\begin{array}{l}\text { Mean } \\
\text { nSNPs }\end{array}$ & $\begin{array}{c}\text { Mode } \\
\text { SNPs per }\end{array}$ & $\begin{array}{c}\text { Max } \\
\text { nSNPs }\end{array}$ \\
\hline 1 & 402 & 0.05 & $25.8 \pm 157.8$ & $11.5 \pm 2.7$ & 13.7 & 2.4 & 2 & 54 \\
\hline 2 & 314 & 0.05 & $23.5 \pm 56.3$ & $12.2 \pm 1.2$ & 14.1 & 2.4 & 2 & 12 \\
\hline 3 & 222 & 0.04 & $20.2 \pm 47.8$ & $12.1 \pm 1.1$ & 13 & 2.3 & 2 & 13 \\
\hline 4 & 186 & 0.065 & $31.4 \pm 210.0$ & $10.9 \pm 2.8$ & 13.6 & 2.4 & 2 & 39 \\
\hline 5 & 118 & 0.06 & $30 \pm 142.3$ & $10.2 \pm 3.5$ & 14 & 2.6 & 2 & 39 \\
\hline 6 & 104 & 0.04 & $14.4 \pm 22.3$ & $10.4 \pm 0.6$ & 13.5 & 2.2 & 2 & 5 \\
\hline 7 & 121 & 0.125 & $37.5 \pm 220.1$ & $12.2 \pm 2.7$ & 18.1 & 2.5 & 2 & 31 \\
\hline 8 & 92 & 0.17 & $53.1 \pm 249.1$ & $12.5 \pm 4.1$ & 21.2 & 3.1 & 2 & 31 \\
\hline 9 & 72 & 0.04 & $13.5 \pm 17.4$ & $12 \pm 1.0$ & 13.8 & 2.2 & 2 & 10 \\
\hline 10 & 94 & 0.06 & $12.6 \pm 17.09$ & $9.5 \pm 0.7$ & 16.6 & 2.2 & 2 & 6 \\
\hline 11 & 95 & 0.14 & $28.4 \pm 118.5$ & $8.2 \pm 2.5$ & 20.6 & 2.6 & 2 & 21 \\
\hline 12 & 99 & 0.07 & $13.2 \pm 25.7$ & $9.8 \pm 1.4$ & 18.5 & 2.3 & 2 & 15 \\
\hline 13 & 93 & 0.08 & $14.8 \pm 23.0$ & $10.6 \pm 1.1$ & 19.2 & 2.3 & 2 & 8 \\
\hline 14 & 69 & 0.09 & $19.34 \pm 41.4$ & $8.9 \pm 2.1$ & 18.5 & 2.7 & 2 & 16 \\
\hline 15 & 89 & 0.11 & $16.1 \pm 21.0$ & $10.3 \pm 1.3$ & 22.6 & 2.5 & 2 & 9 \\
\hline 17 & 66 & 0.11 & $16.9 \pm 28.5$ & $7.5 \pm 1.9$ & 21.5 & 2.7 & 2 & 12 \\
\hline 18 & 58 & 0.06 & $11.5 \pm 22.6$ & $8.6 \pm 0.9$ & 15.2 & 2.2 & 2 & 9 \\
\hline 19 & 53 & 0.11 & $20 \pm 49.4$ & $8.9 \pm 1.8$ & 16.5 & 2.6 & 2 & 13 \\
\hline 20 & 125 & 0.11 & $12.3 \pm 36.4$ & $6.5 \pm 2.2$ & 20.7 & 2.4 & 2 & 25 \\
\hline 21 & 63 & 0.08 & $8.9 \pm 14.8$ & $6.1 \pm 1.2$ & 19.5 & 2.3 & 2 & 10 \\
\hline 22 & 16 & 0.08 & $21.6 \pm 40.7$ & $6.9 \pm 2.8$ & 16.9 & 3.2 & 2 & 12 \\
\hline 23 & 26 & 0.06 & $12.4 \pm 32.3$ & $6.2 \pm 1.4$ & 11.4 & 2.3 & 2 & 9 \\
\hline 24 & 34 & 0.04 & $8.02 \pm 20.0$ & $3.7 \pm 1.7$ & 11.4 & 2.3 & 2 & 12 \\
\hline 25 & 13 & 0.04 & $6.2 \pm 5.1$ & $5.1 \pm 0.4$ & 16.6 & 2.2 & 2 & 3 \\
\hline 26 & 32 & 0.07 & $11.1 \pm 28.5$ & $4.9 \pm 1.4$ & 12.5 & 2.5 & 2 & 9 \\
\hline 27 & 27 & 0.07 & $13.6 \pm 27.6$ & $7.2 \pm 1.7$ & 16.4 & 2.7 & 2 & 10 \\
\hline 28 & 41 & 0.09 & $10.5 \pm 29.22$ & $5.2 \pm 1.9$ & 18.7 & 2.6 & 2 & 14 \\
\hline Average $\pm S D$ & $100.9 \pm 89.2$ & $0.08 \pm 0.03$ & $18.8 \pm 10.4$ & $8.8 \pm 2.6$ & $16.6 \pm 3.3$ & $2.5 \pm 0.26$ & $2 \pm 0.0$ & $16.5 \pm 12.2$ \\
\hline
\end{tabular}

*SD - standard deviation.

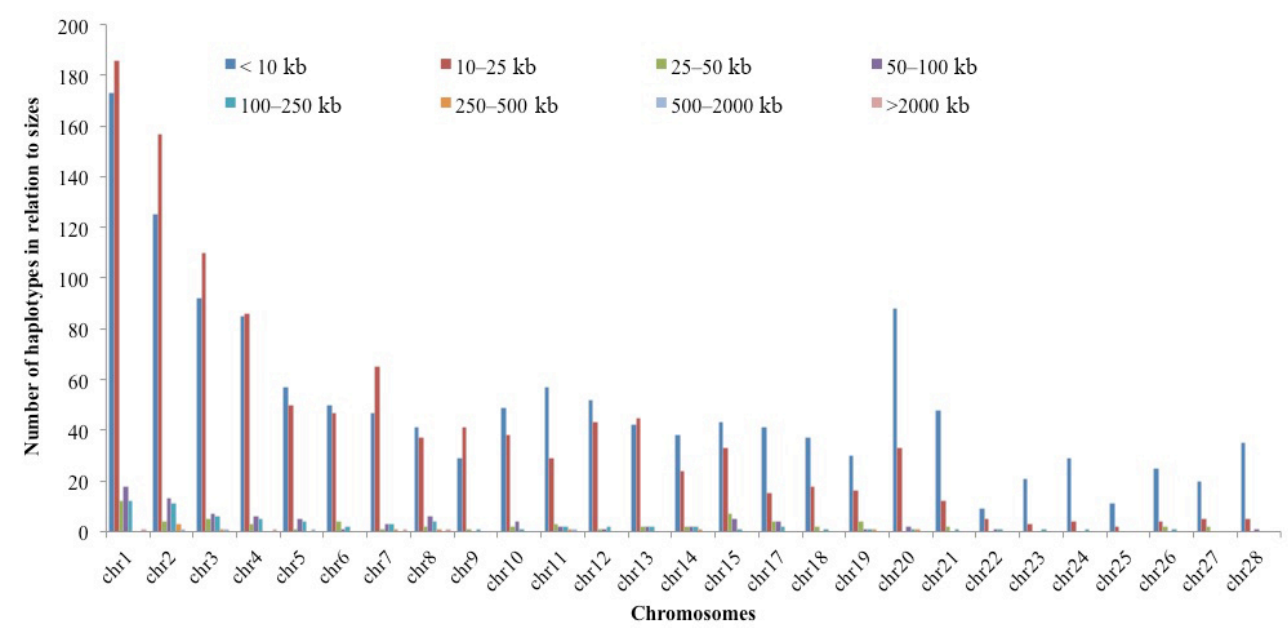

Figure 1. Distribution of haplo-blocks of varying lengths across different chromosomes. 
Table 2. Number of haplo-blocks in relation to their sizes for each population.

\begin{tabular}{lcccr}
\hline & Malawi & South Africa & Zimbabwe & Overall \\
\hline$<10 \mathrm{~kb}$ & 250 & 1132 & 1078 & 1374 \\
$10-25 \mathrm{~kb}$ & 214 & 1023 & 792 & 1115 \\
$25-50 \mathrm{~kb}$ & 44 & 90 & 78 & 64 \\
$50-100 \mathrm{~kb}$ & 56 & 81 & 63 & 83 \\
$100-250 \mathrm{~kb}$ & 63 & 87 & 70 & 71 \\
$250-500 \mathrm{~kb}$ & 13 & 14 & 9 & 10 \\
$500-2000 \mathrm{~kb}$ & 3 & 4 & 4 & 4 \\
$>2000 \mathrm{~kb}$ & 5 & 5 & 5 & 4 \\
\hline
\end{tabular}

\section{Haplotype distribution per population}

Fewer haplo-blocks were observed in populations from Malawi (649) than Zimbabwe (2104) and South Africa (2442) (Table 3). Genome coverage was the lowest in Malawi (39 Mbp) and highest in Zimbabwe (54.5 Mbp) and South Africa (64.4 Mbp). The average block length was highest in populations from Malawi $(60.7 \mathrm{~kb})$, and those from Zimbabwe (25.9 kb) and South Africa $(26.4 \mathrm{~kb})$ were of the same magnitude (Table 3). The percentage of SNPs forming blocks was low in all populations, and ranged from $5 \%$ in Malawi to $14.1 \%$ in South Africa (Table 3).

Table 3. Characteristic of haplo-blocks structure for Malawi, South Africa, and Zimbabwe.

\begin{tabular}{lccr}
\hline & Malawi & South Africa & Zimbabwe \\
\hline Number of blocks & 649 & 2442 & 2104 \\
Genome coverage $(\mathrm{Mbp})$ & 39 & 64.4 & 54.5 \\
Mean block length $(\mathrm{kb}) \pm$ SD & $60.7 \pm 239.6$ & $26.4 \pm 132.9$ & $25.9 \pm 140.5$ \\
Median block length $(\mathrm{kb}) \pm$ SD & $13.6 \pm 239.6$ & $10.8 \pm 132.9$ & $9.7 \pm 140.5$ \\
SNPs (\%) & 5.0 & 14.1 & 11.5 \\
Mean nSNPs & $3.5 \pm 3.9$ & $2.6 \pm 2.7$ & $2.6 \pm 2.7$ \\
Mode SNPs per block & 2 & 55 & 2 \\
Max nSNPs & 49 & & 60 \\
\hline
\end{tabular}

\section{Haplotype sharing between populations}

Zimbabwean and South African chicken populations shared a relatively large number of haplo-blocks (2689 haplo-blocks) whilst Zimbabwean and Malawian populations shared fewer haplo-blocks (2325; Table 4). The proportion of haplo-blocks shared among populations was lower than the total number of 2792 haplo-blocks observed in the overall population. In terms of unique haplotypes, Malawi had 103, Zimbabwe 256, and South Africa 467.

Table 4. Haplo-block sharing between populations (unique haplotypes on the diagonal).

\begin{tabular}{|c|c|c|c|}
\hline & Malawi & South Africa & Zimbabwe \\
\hline Malawi & 103 & - & \\
\hline South Africa & 2536 & 467 & - \\
\hline Zimbabwe & 2325 & 2689 & 256 \\
\hline
\end{tabular}

\section{Haplotype diversity and characteristics}

Haplotype diversity was analyzed on chromosome 8 due to its high LD (Khanyile et al., 2015) and numerous and long haplo-blocks that cover a larger section of the genome compared 
to those on the other chromosomes. On this chromosome, haplo-blocks less than $20 \mathrm{~kb}$ in size were observed on regions 1 and 3. Region 2 had large haplo-blocks (Figure 2) across populations. Malawi populations contained fewer blocks less than $10 \mathrm{~kb}$ in size compared to those carried by Zimbabwe and South African chickens. Region 3 exhibited similar patterns of haplo-block structure across all three populations, with those from Malawi having few haplo-blocks with gaps in between them, and South Africa and Zimbabwe having a number of large blocks that were close to each other. Haplotype blocks less than $20 \mathrm{~kb}$ in size were observed more frequently in Zimbabwean and South African, than in Malawian chickens (Figure 2). Haplo-blocks larger than $50 \mathrm{~kb}$ in size were observed across all genomic regions considered. Variation was observed between genomic regions within populations. Overall, Zimbabwean and South African chickens shared similar patterns of haplo-blocks across the genomic regions under investigation.

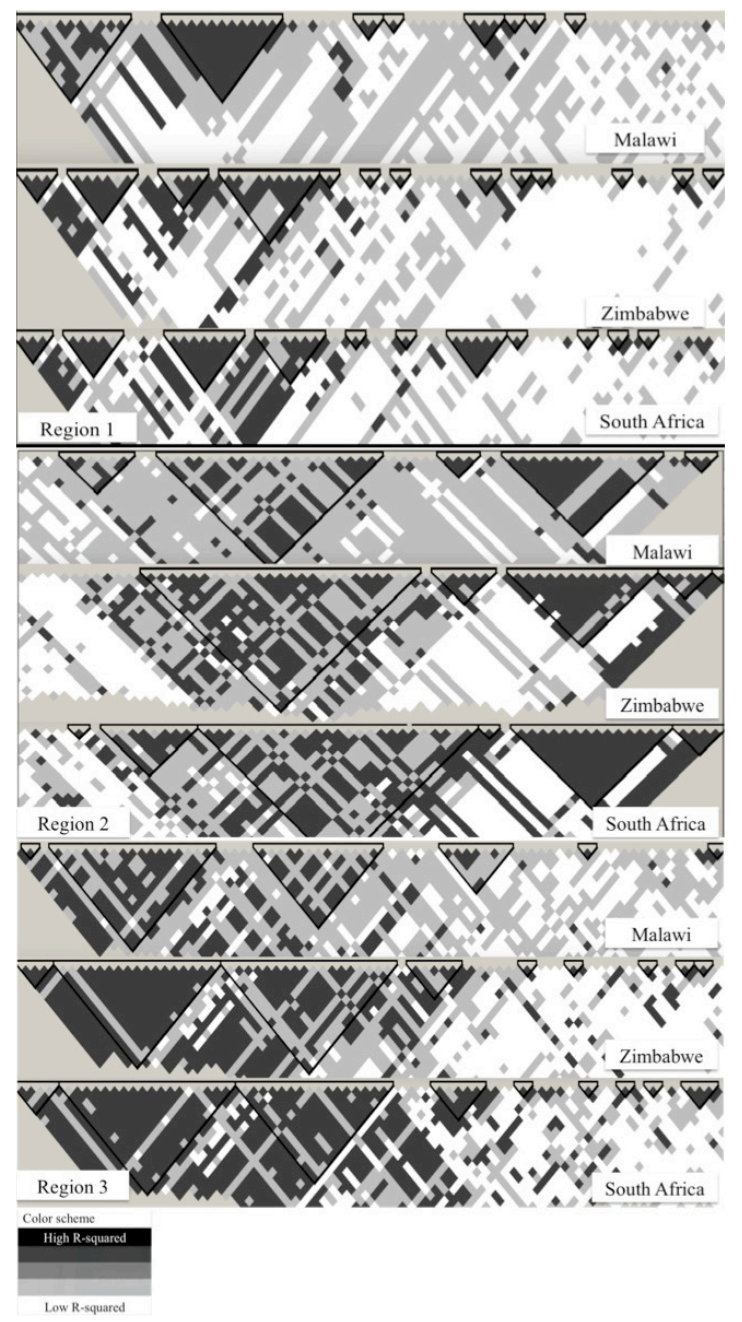

Figure 2. Linkage disequilibrium-bound haplo-block plots of three chromosomal regions on chromosome 8 . Region 1 was found at the edge of thep-arm, region 2 was found close to the centromere of the $p$-arm, and region 3 was found on the q-arm close to the centromere. 
On region 1, South Africa and Zimbabwe shared a similar block pattern with high haplotype diversity within each block compared to populations from Malawi. Zimbabwean populations had a different block pattern to that of Malawian and South African populations on region 2 (Figure 3). All populations had haplo-block sizes greater than $2000 \mathrm{~kb}$ in size (Table 3). These larger blocks contained high haplotype diversity within and between populations (Figure 3).

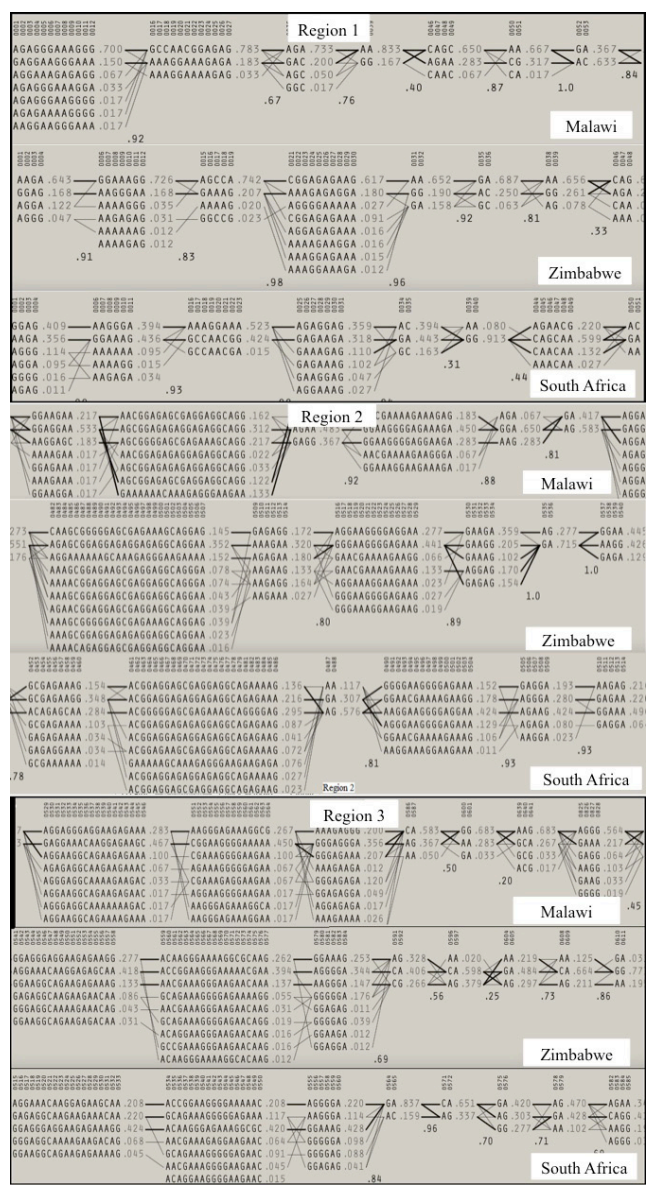

Figure 3. Haplotype plots of chromosome 8 from three chromosomal portions.

Haplotype diversity varied between blocks, and populations from Zimbabwe had high haplotype diversity on block 5 but very low diversity on block 9 (Figure 4). Malawian populations had the highest haplotype diversity, which was high on haplo-block 31 (11 haplotypes), 33 (7 haplotypes), 36 ( 7 haplotypes), and 37 (7 haplotypes), which also corresponded to the size of blocks, which were 477, 197, 486, and $269 \mathrm{~kb}$, respectively (Table 5). Haplo-blocks around the telomeric region did not have QTL matches, except the QTL for spleen percentage, which was found in Zimbabwean and South African chicken populations. The majority of QTLs found were related to body composition traits such as drumstick muscle weight, feed conversion ratio, thigh meat-to-bone ratio, femur bone mineral density, breast muscle weight, body weight (day of first egg), shank weight to length ratio, abdominal fat percentage, and head percentage (Table 5). 


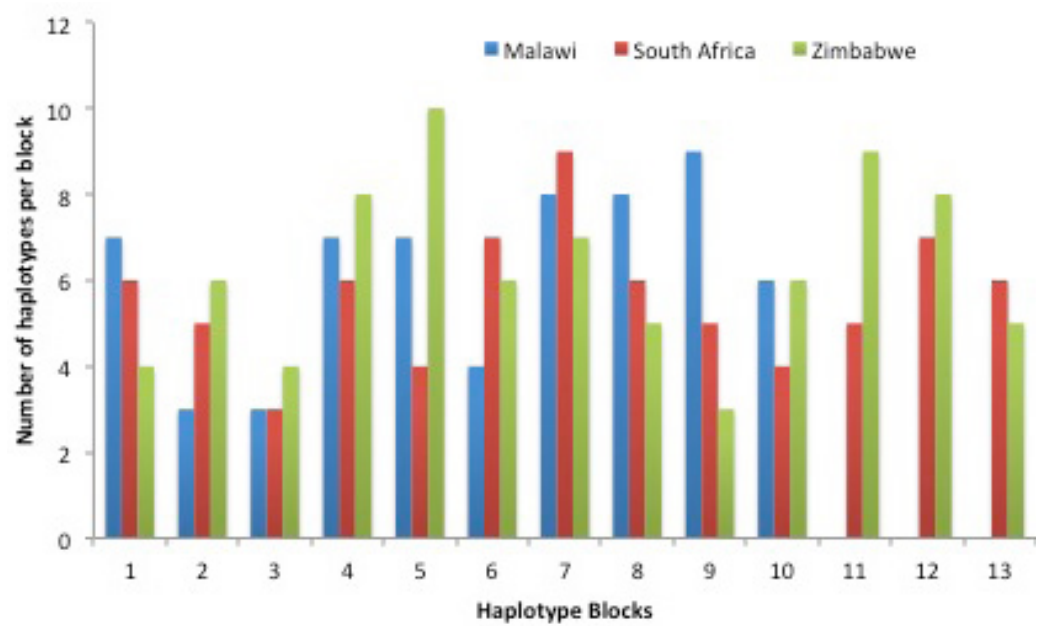

Figure 4. Haplotype diversity within blocks observed on chromosome 8 in extensively raised chicken populations from Malawi, South Africa, and Zimbabwe.

Table 5. Overall haplotype characteristics and QTLs associated with regions covered by haplo-block on chromosome 8 across populations.

\begin{tabular}{|c|c|c|c|c|c|}
\hline Block No. & No. of haplotypes & Location (bp) & Size $(k b)$ & No. of SNPs & QTLs \\
\hline 1 & 5 & $5597-63486$ & 57.89 & 4 & No match \\
\hline 2 & 4 & $129472-221139$ & 91.67 & 4 & SPLP \\
\hline 3 & 6 & $286940-492518$ & 196 & 9 & No match \\
\hline 5 & 5 & $584764-676818$ & 81 & 5 & No match \\
\hline 30 & 6 & 8999746-9090157 & 90.05 & 6 & DSMWT, FBMD, SHKWLR, TW \\
\hline 31 & 11 & $9135643-9613172$ & 477.5 & 25 & DSMWT, TMTBR, FBMD, BMWT, BW, SHKWLR, ABFP, HEADP \\
\hline 32 & 6 & $9639311-9770642$ & 131 & 6 & DSMWT, TMTBR, FBMD, BMWT, BW, SHKWLR, ABFP, HEADP \\
\hline 33 & 7 & $9818354-10011078$ & 192.7 & 15 & DSMWT, TMTBR, FBMD, BMWT, BW, SHKWLR, ABFP, HEADP \\
\hline 34 & 5 & $10016422-10222972$ & 113 & 5 & DSMWT, TMTBR, FBMD, BMWT, BW, SHKWLR, ABFP, HEADP \\
\hline 35 & 4 & $11776856-11841126$ & 100 & 5 & DSMWT, TMTBR, FBMD, BMWT, BW, SHKWLR, ABFP, HEADP \\
\hline 36 & 7 & $11858695-12359447$ & 486 & 17 & DSMWT, TMTBR, FBMD, BMWT, BW, SHKWLR, ABFP, HEADP \\
\hline 37 & 7 & $12429560-12628528$ & 269 & 10 & $\begin{array}{l}\text { DSMWT, THBWT, FCR, CHWID, CRDIG, TIBWT, TW, TIBMD, } \\
\text { TIBPA, TIBSTR, BMWT, BW, GROW, BMWT, DSMWT, SHKWLR, } \\
\text { BMWT, MD, HEADP, WINGWT }\end{array}$ \\
\hline 38 & 6 & $12757762-12838840$ & 81 & 6 & $\begin{array}{l}\text { DSMWT, THBWT, FCR, CHWID, CRDIG, TIBWT, TW, TIBMD, TIBPA, } \\
\text { TIBSTR, BMWT, BW, GROW, SHKWLR, MD, HEADP, WINGWT }\end{array}$ \\
\hline 39 & 5 & $12923972-12987908$ & 63.9 & 5 & $\begin{array}{l}\text { DSMWT, THBWT, FCR, CHWID, CRDIG, TIBWT, TW, TIBMD, TIBPA, } \\
\text { TIBSTR, BMWT, BW, GROW, BMWT, DSMWT, SHKWLR, BMWT, } \\
\text { MD, HEADP, WINGWT }\end{array}$ \\
\hline
\end{tabular}

List of full names of QTLs below: DSMWT = drumstick muscle weight; THBWT = thigh bone weight; FCR = feed conversion ratio; $\mathrm{CHWID}=$ chest width CRDIG = crooked digits; $\mathrm{TIBWT}=$ tibia weight; $\mathrm{TW}$ = tibia width; $\mathrm{TIBMD}=$ tibia marrow diameter; TIBPA = tibia plateau angle; TIBSTR = tibia strength; BMWT = breast muscle weight; BW = body weight (63 days); GROW = growth (21-42 days); SHKWLR = shank weight to length ratio; MD = marek's diseaserelated traits; HEADP = Head percentage; WINGWT = wing weight.

\section{DISCUSSION}

This study sought to determine the structure of haplo-blocks and to identify possible QTLs spanned by haplo-blocks in extensively raised village chicken populations using high-density SNPS. 
Genomic regions have been investigated in a few previous studies that used different commercial chicken lines to analyze the haplo-block structure and haplotype diversity using microsatellite markers, and recently, genome-wide SNP data. As a result, studies such as those by Megens et al. (2009), Qanbari et al. (2010), and Wragg et al. (2012) are available and can be used for comparisons. The haplo-block structure of village chicken populations from Southern Africa should permit understanding of their genomics and provide baseline information for future investigations on haplotype variation in association with phenotypic traits of interest.

Although the number of haplo-blocks was high on the macro-chromosomes, the chromosomal coverage was low, indicating the presence of many short haplo-blocks in all populations. The average length of haplo-blocks in these regions was $26 \mathrm{~kb}$. Multiple short haploblocks imply that many recombination events occurred that broke up the historical ancestral blocks into smaller segments. These results were expected from outbred populations with high genetic diversity that has not been subjected to artificial selection pressures for specific production traits (Wragg et al., 2012). The average median block length (Table 1) observed in this study was 8.8 $\mathrm{kb}$, and is comparable to that reported in chickens (Megens et al., 2009), wild dogs (Zhao et al., 2003; Lindblad-Toh et al., 2005), pigs (Amaral et al., 2008), and humans (Gabriel et al., 2002; Wall and Pritchard 2003). The number of SNPs forming blocks was above 25 in some chromosomes, including chromosomes 1, 4, 5, 7, and 8. These genomic regions have long stretches of haploblocks, and they should be further investigated for association with morphological or quantitative traits that could be spanned by these blocks. A high proportion of haplo-blocks were less than 10 $\mathrm{kb}$ in size, with some falling between 10 and $25 \mathrm{~kb}$ across all populations. Very few haplo-blocks were more than $500 \mathrm{~kb}$ in size. Megens et al. (2009) reported haplo-blocks less than $10 \mathrm{~kb}$ long on targeted genomic regions on four chromosomes (chromosome 1, 2, 26, and 27), from both macro- and micro-chromosomes. Haplo-blocks less than $10 \mathrm{~kb}$ in size were observed in regions of low LD ( 0.2) in the presumably outbred populations (Megens et al., 2009). A large proportion of haplo-blocks occurred at a frequency greater than $20 \%$ in the overall population (Figure 1). These moderately prevalent haplo-blocks can be used to assess haplotype diversity and genetic variation within and between populations.

The number of observed haplo-blocks varied between populations. Malawian populations had the lowest number of haplo-blocks compared to those from South Africa and Zimbabwe. This could have been caused by the sampling structure, in that one ecotype of chickens was obtained from Malawi whereas three ecotypes country were used for South Africa and Zimbabwe. The number of haplo-blocks in Malawi was similar to that observed by Wragg et al. (2012) in traditional and village chicken populations. The number of haplo-blocks in populations from South Africa and Zimbabwe was similar to that observed by Qanbari et al. (2010) in commercial lines (broilers and layers). Overall, a high number of haplo-blocks was expected from the Southern African populations, as these chickens are diversely outbred and have not been selected for any specific traits. Findings from this study show that South African and Zimbabwean chicken populations have accumulated more haplo-blocks compared to Malawi populations. These blocks are small in size (less than $25 \mathrm{~kb}$ ) suggesting higher effective population sizes and low levels of inbreeding in South African and Zimbabwean chickens compared to those from Malawi.

The high level of haplo-block sharing determines the transferability of genetic parameters between populations (Megens et al., 2009). Haplotype sharing in this study varied with a considerable number of haplo-blocks being shared between populations. Chickens from Malawi shared more haplo-blocks with other populations and only a few haplo-blocks were unique to populations from this country. Further analysis of the size of haplotypes shared, their genomic 
content, and the frequency between populations can provide more insight into genomic regions that span economically important traits. Variation in the number of unique haplo-blocks within a population indicates independent genomic sub-structuring and evolution of populations. There is a need to identify the unique and shared genomic regions found between populations with the aim of associating haplotypes with adaptive traits in these extensively reared populations.

Haplo-blocks observed in this study had 10- to 16-fold lower genome coverage in comparison to that reported by Qanbari et al. (2010), where blocks covered more than $300 \mathrm{Mbp}$ of the $1.05 \mathrm{Gbp}$ chicken genome in both broilers and layers, respectively. The median block length and mode SNPs per block were similar to those observed in traditional chicken populations by Wragg et al. (2012). These results indicate that the genomes of extensively raised chickens still resemble the ancestral block length, which is similar to findings in other species such as dogs, irrespective of their different domestication histories and demographic evolutionary processes (Lindblad-Toh et al., 2005). The number of SNPs forming a block was low in comparison to that observed in studies conducted on commercial lines (Qanbari et al., 2010). Denser SNP panels, such as the 600K SNP chip, would probably improve the number and proportion of SNPs that form haplo-blocks and will also improve the LD fine mapping of QTLs and association studies in these extensively raised chicken populations.

On the regions investigated, QTLs have been found to be associated with traits associated with growth and body composition such as body weight, muscle weight, tibia, wings, and thigh size. Results indicate the importance of such traits in village chicken populations. Growth and body composition traits could allow village chicken populations to adapt to fluctuating and scarce feed in the extensive management systems under which most of these birds are reared. Village chickens are known to be slow growers (Muchadeyi et al., 2007b), a characteristic that might reduce the risk of them dying during periods of feed shortage. The rate of growth and body weight might therefore be associated with the observed haplo-block structure in these chicken populations. The presence of known QTLs related to body composition on the observed haplo-blocks indicates the possibility of LD fine mapping of QTLs in extensively raised chicken populations using genome-wide high density SNP panels such the Illumina iSelect chickens SNP60K array. Further investigation of chromosomal regions flanked by large haplo-blocks and QTL association in other regions can be useful for genomics assisted selection to improve fertility, growth, and feed conversion efficiency.

Overall, this study revealed low haplo-block genome coverage on most chromosomes due to the existence of multiple small blocks. Such a scenario might limit the use of haplo-blocks in QTL mapping in certain genomic regions in extensively raised chicken. Regions that were covered by long haplo-blocks spanned QTL regions associated with economically important traits and could be useful in gene mapping. The observed haplotype diversity on chromosome 8 and the difference in haplo-block structure within and between populations suggests that genetic diversity was shaped by differences in agro-ecological and farming system factors in Southern African village chicken populations.

\section{Conflicts of interest}

The authors declare no conflict of interest.

\section{AKNOWLEDGMENTS}

Genotyping of samples was funded by the Agricultural Research Council-Biotechnology 
Platform (ARC-BTP) while Mr. K. S. Khanyile held fellowships from the ARC-Professional Development Program, National Research Foundation of South Africa and the University of KwaZulu-Natal postgraduate program.

\section{REFERENCES}

Amaral AJ, Megens HJ, Crooijmans RP, Heuven HC, et al. (2008). Linkage disequilibrium decay and haplotype block structure in the pig. Genetics 179: 569-579.

Barrett JC, Fry B, Maller J and Daly MJ (2005). Haploview: analysis and visualization of LD and haplotype maps. Bioinformatics 21: 263-265.

Crawford DC and Nickerson DA (2005). Definition and clinical importance of haplotypes. Annu. Rev. Med. 56: 303-320.

Ensembl (2013). Chicken Genome. Available at [http://www.ensembl.org/Gallus_gallus/Location/Genome]. Accessed October, 2013.

Gabriel SB, Schaffner SF, Nguyen H, Moore JM, et al. (2002). The structure of haplotype blocks in the human genome. Science 296: 2225-2229.

International Chicken Genome Sequencing Consortium (2004). Sequence and comparative analysis of the chicken genome provide unique perspectives on vertebrate evolution. Nature 432: 695-716.

Khanyile KS, Dzomba EF and Muchadeyi FC (2015). Population genetic structure, linkage disequilibrium and effective population size of conserved and extensively raised chicken populations of Southern Africa. Front. Genet. 6: 13.

Li MH and Merilä J (2011). Population differences in levels of linkage disequilibrium in the wild. Mol. Ecol. 20: 2916-2928.

Lindblad-Toh K, Wade CM, Mikkelsen TS, Karlsson EK, et al. (2005). Genome sequence, comparative analysis and haplotype structure of the domestic dog. Nature 438: 803-819.

Megens HJ, Crooijmans RP, Bastiaansen JW, Kerstens HH, et al. (2009). Comparison of linkage disequilibrium and haplotype diversity on macro-and microchromosomes in chicken. BMC Genet. 10: 86.

Mtileni BJ, Muchadeyi FC, Maiwashe A, Groeneveld E, et al. (2011). Genetic diversity and conservation of South African indigenous chicken populations. J. Anim. Breed. Genet. 128: 209-218.

Muchadeyi FC, Sibanda S, Kusina NT, Kusina J, et al. (2005). Village chicken flock dynamics and the contribution of chickens to household livelihoods in a smallholder farming area in Zimbabwe. Trop. Anim. Health. Prod. 37: 333-344.

Muchadeyi FC, Weigend S, Wollny C and Makuza S (2006). Use of microsatellites and mtDNA to assess genetic diversity within and between Zimbabwe chicken eco-types. In: International Research on Food Security, University of Bonn.

Muchadeyi FC, Eding H, Wollny CB, Groeneveld E, et al. (2007a). Absence of population substructuring in Zimbabwe chicken ecotypes inferred using microsatellite analysis. Anim. Genet. 38: 332-339.

Muchadeyi F, Wollny C, Eding H, Weigend S, et al. (2007b). Variation in village chicken production systems among agroecological zones of Zimbabwe. Trop. Anim. Health. Prod. 39: 453-461.

Purcell SN, Todd-Brown BK, Thomas L, Ferreira M, et al. (2007). Plink: a toolset for whole-genome association and populationbased linkage analysis. Available at [http://pngu.mgh.harvard.edu/purcell/plink/]. Accessed March, 2012.

Qanbari S, Hansen M, Weigend S, Preisinger R, et al. (2010). Linkage disequilibrium reveals different demographic history in egg laying chickens. BMC Genet. 11: 103.

Tishkoff SA and Verrelli BC (2003). Role of evolutionary history on haplotype block structure in the human genome: implications for disease mapping. Curr. Opin. Genet. Dev. 13: 569-575.

Wall JD and Pritchard JK (2003). Haplotype blocks and linkage disequilibrium in the human genome. Nat. Rev. Genet. 4: 587-597.

Wragg D, Mwacharo JM, Alcalde JA, Hocking PM, et al. (2012). Analysis of genome-wide structure, diversity and fine mapping of Mendeliantraits in traditional and village chickens. Heredity 109: 6-18.

Zhao H, Pfeiffer R and Gail MH (2003). Haplotype analysis in population genetics and association studies. Pharmacogenomics 4: 171-178. 